\title{
Quantification of Ventricular Repolarization Fluctuations in Patients With Myocardial Infarction
}

\author{
Martin Schmidt ${ }^{1}$, Robin Dunker ${ }^{1}$, Hagen Malberg ${ }^{1}$, Sebastian Zaunseder ${ }^{2}$ \\ ${ }^{1}$ Institute of Biomedical Engineering, TU Dresden, Dresden, Germany \\ ${ }^{2}$ Faculty of Information Technology, University of Applied Science and Arts Dortmund, Dortmund, \\ Germany
}

\begin{abstract}
The objective of this study was to quantify ventricular repolarization fluctuations in patients with myocardial infarction (MI) by a novel ECG waveform morphology based $Q T$ interval variability (QTV) measure. We analyzed recordings of 79 MI patients and 69 healthy control subjects included in the Physikalisch-Technische Bundesanstalt diagnostic ECG database. To characterize the QT interval waveform, we employed two-dimensional signal warping (2DSW). Based on the two-dimensional template adaptation to every beat, a novel parameter QTfluc has been developed to take into account complex QT interval's waveform fluctuations in time and amplitude. To demonstrate the power of QTfluc we (1) compared MI patients and healthy subjects and (2) examined the stability of various QTV measures including QTfluc in relation to QT interval boundary shifts. A comparison of QTfluc with standard QTV measures showed a significant improvement (effect size increased up to $60 \%$ ) in discriminating between MI patients and healthy subjects. QT interval boundary shifts showed significant less impact (by factor 10) on the stability of QTfluc in comparison to standard QTV measures. The proposed measure showed significant improved characterization of ventricular repolarization lability in MI patients. Moreover, QTfluc showed more stable characteristics and is less dependent on QT interval boarders.
\end{abstract}

\section{Introduction}

QT interval variations of the surface electrocardiogram (ECG) reflect beat-to-beat fluctuations of the ventricular repolarization. Several studies have shown that temporal repolarization lability as indicated by an increased QT interval variability (QTV) is associated with cardiac mortality $[1,2]$. However, measuring subtle beat-to-beat changes in QT interval remains challenging. Although novel QTV techniques have improved sensitivity and robustness $[3,4]$, conventional QTV measures still lack in insightful QTV description [1].

Alongside the improved QTV measurement, new techniques are able to capture the entire QT interval waveform morphology [4,5] and thus, in contrast to standard parameters based on QT interval time and its variability, offer a new feature set for the characterization of the ventricular repolarization. However, standard QTV parameter are predominantly used in most QTV studies [1].

In this study we propose a novel ECG waveform morphology based QTV measure for the characterization of the ventricular repolarization lability and employ it in patients with myocardial infarction (MI). Based on the twodimensional waveform characterization of every beat, a novel fluctuation parameter denoted as QTfluc has been developed. QTfluc takes into account complex QT interval's waveform fluctuations instead of its length only. To demonstrate the power of QTfluc we (1) compared MI patients and healthy subjects and (2) examined the stability of QTV measures in relation to QT interval boundary shifts, i.e. we varied the interval length over which morphological changes were expected to affect QTfluc.

\section{Material and Methods}

\subsection{Data Material}

We used data originating from the PhysikalischTechnische Bundesanstalt (PTB) diagnostic ECG database [6] to measure pathophysiological variations in QT interval. The PTB database consists of 79 patients with myocardial infarction (MI) (22 female, age: $63 \pm$ 12 years; 57 males, age: $57 \pm 10$ years) and 69 healthy control subjects (17 females, age: $42 \pm 18$ years; 52 males, age: $40 \pm 13$ years). Standard 12-lead ECGs were recorded at $1000 \mathrm{~Hz}$ during rest for about $2 \mathrm{~min}$. Previous studies reported higher QTV in MI patients compared to healthy subjects $[1,4,7]$. We performed our analysis in lead I because of the highest $\mathrm{T}$ wave amplitude and thus maximized signal-to-noise ratio [8]. 


\subsection{Feature Extraction}

To capture beat-to-beat waveform fluctuations in ECG, we used iterative two-dimensional signal warping (i2DSW) $[4,9]$. The method is capable to account for subtle inhomogeneous shape variations of the ECG waveform in time and amplitude. i2DSW automatically generates a template beat with common features of interest (e.g. the PQ, QRS or QT intervals or amplitude related information) based on ensemble averaging of appropriate beats. To adapt the template, a 2D mesh of warping points is superimposed. By shifting warping points in time- and amplitude-direction and minimizing the Euclidean distance between template and the beat under consideration, the optimal template adaptation is calculated. From the adapted template, changes of annotated points can be tracked from beat to beat in time and amplitude. Next to the adapted template we used QT intervals and T wave amplitudes for further analysis.

We applied automatic beat rejection [4] to exclude noisy heart beats. To quantify QTV and to compare the novel QTfluc against standard parameters, we calculated standard deviations of QT intervals (SDQT) and to characterize $\mathrm{T}$ wave amplitude variations we calculated $\mathrm{T}$ wave alternans (TWA) by the approach of Nearing et al. [10].

\subsection{Quantification of QT Fluctuations}

In order to characterize the fluctuations of the QT interval waveform in time $x$ and amplitude $y$, we developed the parameter QTfluc based on the template adapted to every beat by i2DSW. Representing every $M$ adopted templates of length $N$, the Matrix $\mathbf{B}_{\mathbf{x}}$ was constructed as follows:

$$
\begin{aligned}
\mathbf{B}_{\mathbf{x}}= & {\left[\begin{array}{cccc}
x_{1,1}-\widetilde{x}_{1} & x_{1,2}-\widetilde{x}_{2} & \ldots & x_{1, N}-\widetilde{x}_{N} \\
x_{2,1}-\widetilde{x}_{1} & x_{2,2}-\widetilde{x}_{2} & \ldots & x_{2, N}-\widetilde{x}_{N} \\
\vdots & \vdots & \vdots & \vdots \\
x_{M, 1}-\widetilde{x}_{1} & x_{M, 2}-\widetilde{x}_{2} & \ldots & x_{M, N}-\widetilde{x}_{N}
\end{array}\right] } \\
= & {\left[\begin{array}{cccc}
X_{1,1} & X_{1,2} & \ldots & X_{1, N} \\
X_{2,1} & X_{2,2} & \ldots & X_{2, N} \\
\vdots & \vdots & \vdots & \vdots \\
X_{M, 1} & X_{M, 2} & \ldots & X_{M, N}
\end{array}\right] }
\end{aligned}
$$

with

$$
\widetilde{x}_{n}= \begin{cases}x_{\frac{N+1}{2}} & \text { for odd } N \\ \frac{1}{2}\left(x_{\frac{N}{2}}+x_{\frac{N}{2}+1}\right) & \text { for even } N\end{cases}
$$

where $x_{m, 1} \ldots x_{m, N}$ are the $x$ coordinates for the adopted Template with $m=[1 \ldots M], \widetilde{x}_{n}$ is the median for $x$ coordinate with $n=[1 \ldots N]$, and $X_{m, n}$ is the median cor- rected $x$ coordinate. Based on $\mathbf{B}_{\mathbf{x}}$ we calculated the fluctuation matrix

$$
\mathbf{F}_{\mathbf{x}}=\left[\begin{array}{llll}
\sigma_{x, 1} & \sigma_{x, 2} & \ldots & \sigma_{x, N}
\end{array}\right]
$$

where $\sigma_{x, n}$ represents the fluctuation of coordinate $n$ in $x$ calculated by

$$
\sigma_{x, n}=\sqrt{\frac{1}{M-1} \sum_{i=1}^{M}\left(X_{i, n}-\bar{X}_{n}\right)^{2}}
$$

with

$$
\bar{X}_{n}=\frac{\sum_{i=1}^{M} X_{i, n}}{M} .
$$

The so-called parameter QTfluc $\mathrm{X}$ is the median fluctuation $\sigma_{x, n}$ of the matrix $\mathbf{F}_{\mathbf{x}}$ calculated as follows:

$$
\text { QTfluc }_{\mathbf{X}}=\widetilde{\mathbf{F}_{\mathbf{X}}}= \begin{cases}\sigma_{x, \frac{N+1}{2}} & \text { for odd } N \\ \frac{1}{2}\left(\sigma_{x, \frac{N}{2}}+\sigma_{x, \frac{N}{2}+1}\right) & \text { for even } N\end{cases}
$$

QTfluc $_{\mathrm{X}}$ normalized by the mean QT interval $Q T_{\text {mean }}$ and scaled by scaling factor $\omega$ is calculated as

$$
\text { QTfluc }_{X, \text { norm }}=\frac{1}{Q T_{\text {mean }}} \cdot \text { QTfluc }_{\mathrm{X}}
$$

Thus, QTfluc $\mathrm{X}$, is a complex measure that captures the temporal beat-to-beat variability of a set of points. Analogously to the calculation of the QT fluctuations in time $x$ by QTfluc $_{X}$, the calculation of the fluctuations in amplitude is done resulting in $\mathrm{QTfluc}_{\mathrm{Y}}$ and

$$
\text { QTfluc }_{\mathrm{Y}, \mathrm{norm}}=\frac{1}{\bar{T}_{a m p}} \cdot \text { QTfluc }_{\mathrm{Y}}
$$

where $\bar{T}_{a m p}$ is the median $\mathrm{T}$ wave amplitude.

QTfluc represents the QT interval waveform fluctuations in time and amplitude and is based on the combination of

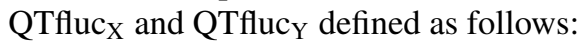

$$
\text { QTfluc }=\sqrt{\text { QTfluc }_{\mathrm{X}, \text { norm }}{ }^{2}+\text { QTfluc }_{\mathrm{Y}, \text { norm }}{ }^{2}}
$$

We scaled the normalized QTfluc parameters by a factor of 1000 to achieve a comparable domain to nonnormalized and standard parameters and thus a better visualization.

\subsection{Statistical Analysis}

SDQT, TWA, and non-normalized and normalized QTfluc parameters have been tested for standard normal 
Table 1. Comparison of healthy controls and patients with myocardial infarction (MI) of the PTB diagnostic ECG database by QT interval variability and $\mathrm{T}$ wave alternans versus QT fluctuation in time and amplitude (median \pm interquartile range). Statistical evaluations were performed with a Wilcoxon rank sum test ( $P$ value) and effect size $r$.

\begin{tabular}{lcccc}
\hline \hline & healthy & MI & $P$ & $r$ \\
\hline SDQT, ms & $2.77 \pm 2.10$ & $3.87 \pm 4.46$ & $<0.001$ & 0.28 \\
QTfluc $_{X}$, ms & $1.38 \pm 0.87$ & $2.46 \pm 5.86$ & $<0.001$ & 0.39 \\
QTfluc $_{X, \text { norm }}, 1$ & $3.63 \pm 2.58$ & $6.17 \pm 13.35$ & $<0.001$ & 0.37 \\
\hline TWA, $\mu \mathrm{V}$ & $11.21 \pm 22.78$ & $10.44 \pm 15.92$ & 0.207 & 0.11 \\
QTfluc $_{Y}, \mu \mathrm{V}$ & $8.71 \pm 7.27$ & $11.48 \pm 9.57$ & $<0.050$ & 0.21 \\
QTfluc $_{Y, \text { norm }}, 1$ & $4.96 \pm 3.72$ & $11.94 \pm 16.88$ & $<0.001$ & 0.41 \\
\hline QTfluc, 1 & $6.37 \pm 4.40$ & $16.58 \pm 34.80$ & $<0.001$ & 0.45 \\
\hline
\end{tabular}

$\mathrm{X}$, time domain; Y, amplitude domain; SDQT, standard deviation of QT intervals; QTfluc $\mathrm{X}$, QT fluctuation in time; QTfluc $\mathrm{X}$,norm, normalized QT fluctuation in time; TWA, T wave alternans; QTfluc ${ }_{Y}$, QT fluctuation in amplitude; QTfluc ${ }_{\text {Ynorm }}$, normalized QT fluctuation in amplitude; QTfluc, normalized QT fluctuation in time and amplitude.

distribution using the Kolmogorov-Smirnov test. Group comparisions between healthy control subjects and MI patients have been done with the Wilcoxon rank sum test and quantified by the Wilcoxon effect size $r$. We compared the effect sizes for fluctuations in time and amplitude with the corresponding standard measures to demonstrated the improvements in group discrimination. To analyze the stability of QTfluc and standard measures in relation to QT interval boundary shifts, we varied the end of the QT interval boarder (QTend) by $\pm 10 \mathrm{~ms}$ to its original annotation. For each QTend shift we calculated the considered measures and quantified the stability by the median range and the coefficient of variation.

\section{Results and Discussion}

Kolmogorov-Smirnov test showed non-standard normal distribution for all analyzed parameters $(P<0.05)$. Consequently, Wilcoxon rank sum test has been used for group comparisons between healthy control subjects and MI patients. Table 1 shows the results of group discrimination for QT fluctuation parameters in time and amplitude in comparison to standard parameters. SDQT, QTfluc $\mathrm{X}_{\mathrm{X}}$

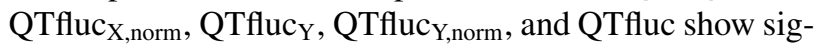
nificant differences between healthy control subjects and MI patients $(P<0.05)$. QTV and QTfluc parameters are increased in Patients with MI compared to healthy subjects. Only TWA does not show this behavior $(P=0.207)$. By comparing SDQT with QTfluc in time, we found an increased effect size $r$ from a small effect $\left(r_{\mathrm{SDQT}}=0.28\right)$ to a medium effect $\left(r_{\mathrm{QTfluc}_{\mathrm{X}}}=0.39 ; r_{\mathrm{QTfluc}} \mathrm{C}_{\mathrm{Xnorm}}=0.37\right)$. In amplitude domain QTfluc showed higher effect sizes $\left(r_{\mathrm{QTfluc}}=0.21 ; r_{\mathrm{QTfluc}}^{\mathrm{X}, \mathrm{norm}}=0.41\right)$ compared to TWA
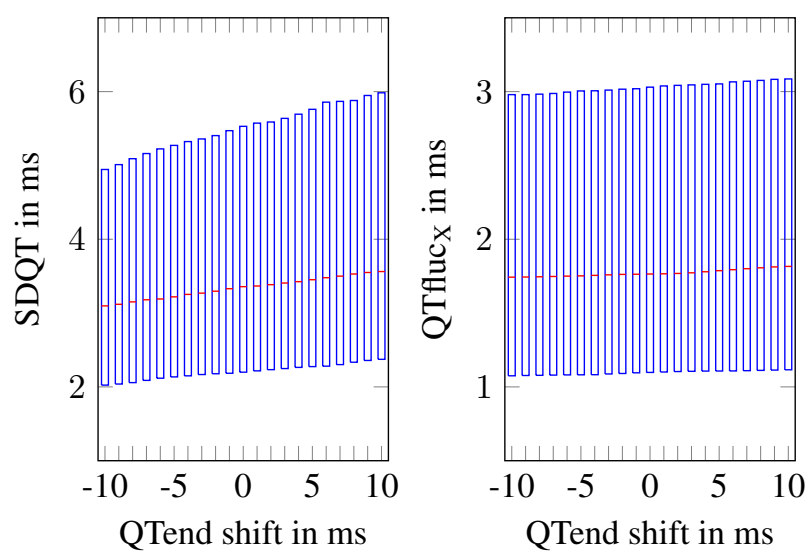

Figure 1. Standard deviation of QT intervals (SDQT) and QT fluctuation in time $\left(\right.$ QTfluc $\left._{X}\right)$ for different delineations of the QT interval end (QTend) generated by shifting of the QTend boundary (QTend shift) in all datasets of the PTB diagnostic ECG database. Values are plotted as median and interquartile range.

$\left(r_{\mathrm{SDQT}}=0.11\right)$. Calculating QTflux in time and amplitude domain showed the highest effect sizes for discriminating between both groups $\left(r_{\mathrm{QTfluc}}=0.45\right)$. Compared to SDQT, the effect size of QTfluc showed a significant improvement group discrimination of up to $60 \%$.

Figure 1 shows the behavior of SDQT and QTfluc ${ }_{X}$ to QTend shifts. SDQT increases the more QTend is shifted forward in time and decreases with negative QTend time

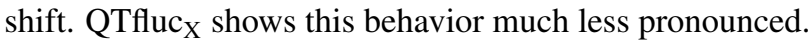
Numerical results are shown in Table 2 by the median range and $\mathrm{CV}$ of the considered parameters. $\mathrm{CV}$ minimized from 0.039 (SDQT) to 0.004 (QTfluc $_{X}$ ) in healthy subjects and from 0.067 to 0.002 in MI patients. We found similar results for the QTfluc ${ }_{X, \text { norm }}$ compared to SDQT. In both groups healthy subjects and MI QTfluc parameters show smaller CVs by factor 10 thus so more stable results regarding the delineation of the QTend.

Our results confirm previous findings that MI patients yield higher myocardial repolarization variability compared to healthy subjects. The introduced measure QTfluc to characterize such fluctuations in ECG shows better separation between groups. Especially in amplitude quantification, the normalization of QTfluc by $\mathrm{T}$ wave amplitude brings an increased group discrimination. This effect could be due to the relationship of QTV and T wave amplitude, which is an result of low signal-to-noise ratio by low $\mathrm{T}$ wave amplitudes [8]. Further investigations will analyze the interaction between QTfluc and the normalization in time and amplitude. By considering fluctuations of the entire waveform, more robust results could be obtained depending on the delineation of the QTend. This confirms the hypothesis that the ventricular repolarization process is 
Table 2. Effect of the QT interval end (QTend) boundary shifting on QT interval variability and QT fluctuation in healthy controls and patients with myocardial infarction (MI) of the PTB's diagnostic ECG database. QTend was shifted between $-10 \mathrm{~ms}$ and $10 \mathrm{~ms}$ in $1 \mathrm{~ms}$ steps. Results are shown as median range and its coefficient of variation (CV).

\begin{tabular}{llclc}
\hline \hline & \multicolumn{2}{c}{ healthy } & \multicolumn{2}{c}{ MI } \\
\cline { 2 - 5 } & median & $\mathrm{CV}$ & median & $\mathrm{CV}$ \\
\hline SDQT, ms & $2.61-2.95$ & 0.0389 & $3.35-4.12$ & 0.0667 \\
QTfluc $_{X}, \mathrm{~ms}$ & $1.37-1.38$ & 0.0038 & $2.45-2.47$ & 0.0023 \\
QTfluc $_{X, \text { norm }}, 1$ & $3.58-3.68$ & 0.0093 & $6.14-6.29$ & 0.0076 \\
\hline
\end{tabular}

X, time domain; SDQT, standard deviation of QT intervals; QTfluc $\mathrm{X}, \mathrm{QT}$ fluctuation in time; QTfluc $\mathrm{X}_{\mathrm{X}, \text { norm }}$, normalized QT fluctuation in time.

highly complex and can be characterized much better by the analysis of the entire morphology of the QT interval than by its duration. In summary, the QTfluc parameter presented here provides a highly sensitive and at the same time more robust ECG-based measure for the characterization of ventricular repolarization. It enables the analysis of the fluctuation in time and amplitude.

\section{Conclusion}

The proposed measure to quantify QT fluctuations shows significant improved characterization of ventricular repolarization lability in MI patients. Moreover, QTfluc shows more stable characteristics and is less dependent on QT interval end delineation. Further investigations will focus on the evaluation of additional datasets and the application to other quasiperiodic biosignals.

\section{Acknowledgments}

This study was partly supported by grants from the European Social Found and the Free State of Saxony (ESF 100339450, "MEDICOS").

\section{References}

[1] Baumert M, Porta A, Vos MA, Malik M, Couderc JP, Laguna P, Piccirillo G, Smith GL, Tereshchenko LG, Volders PGA. QT Interval Variability in Body Surface ECG: Measurement, Physiological Basis, and Clinical Value: Position Statement and Consensus Guidance Endorsed by the European Heart Rhythm Association Jointly with the ESC Working Group on Cardiac Cellular Electrophysiology. Europace June 2016;18(6):925-944.

[2] Schmidt M, Baumert M, Penzel T, Malberg H, Zaunseder S. Nocturnal Ventricular Repolarization Lability Predicts Cardiovascular Mortality in the Sleep Heart Health Study. Am J Physiol Heart Circ Physiol December 2018;316(3):H495H505.
[3] Baumert M, Starc V, Porta A. Conventional QT Variability Measurement vs. Template Matching Techniques: Comparison of Performance Using Simulated and Real ECG. PLoS ONE July 2012;7(7):e41920.

[4] Schmidt M, Baumert M, Porta A, Malberg H, Zaunseder S. Two-Dimensional Warping for One-Dimensional SignalsConceptual Framework and Application to ECG Processing. IEEE Trans Signal Process November 2014; 62(21):5577-5588.

[5] Ramírez J, Orini M, Tucker JD, Pueyo E, Laguna P. Variability of Ventricular Repolarization Dispersion Quantified by Time-Warping the Morphology of the T-Waves. IEEE Transactions on Biomedical Engineering July 2017; 64(7):1619-1630. ISSN 1558-2531. Conference Name: IEEE Transactions on Biomedical Engineering.

[6] Bousseljot R, Kreiseler D, Schnabel A. Nutzung der EKGSignaldatenbank CARDIODAT der PTB über das Internet. Biomed Tech Eng 2009;40(s1):317-318.

[7] Hasan MA, Abbott D, Baumert M. Beat-to-Beat QT Interval Variability and T-Wave Amplitude in Patients with Myocardial Infarction. Physiol Meas 2013;34(9):1075. ISSN 0967-3334.

[8] Schmidt M, Baumert M, Malberg H, Zaunseder S. T Wave Amplitude Correction of QT Interval Variability for Improved Repolarization Lability Measurement. Front Physiol 2016;7.

[9] Schmidt M, Baumert M, Malberg H, Zaunseder S. Iterative Two-Dimensional Signal Warping-Towards a Generalized Approach for Adaption of One-Dimensional Signals. Biomed Signal Process Control May 2018;43:311-319.

[10] Nearing BD, Verrier RL. Modified Moving Average Analysis of T-Wave Alternans to Predict Ventricular Fibrillation with High Accuracy. Journal of Applied Physiology 2002; 92(2):541-549.

Address for correspondence:

Martin Schmidt

Institute of Biomedical Engineering, TU Dresden

Fetscherstraße 29, 01307 Dresden, Germany

martin_schmidt@tu-dresden.de 